\title{
Dscam and DSCAM: complex genes in simple animals, complex animals yet simple genes
}

\author{
Dietmar Schmucker ${ }^{1,2,5}$ and Brian Chen $^{3,4}$ \\ ${ }^{1}$ Department of Cancer Biology, Dana-Farber Cancer Institute, Boston, Massachusetts 02115, USA; ${ }^{2}$ Department of \\ Neurobiology, Harvard Medical School, Boston, Massachusetts 02115, USA; ${ }^{3}$ Centre for Research in Neuroscience, McGill \\ University Health Centre, Montreal, Quebec H3G 1A4, Canada; ${ }^{4}$ Montreal General Hospital, Montreal, Quebec H3G 1A4, \\ Canada
}

Cadherins and the immunoglobulin (Ig) proteins give rise to a multitude of surface receptors, which function as diverse cell adhesion molecules (CAMs) or signaltransducing receptors. These functions are often interdependent, and rely on a high degree of specificity in homophilic binding as well as heterophilic interactions. The Drosophila receptor Dscam is an exceptional example of homophilic binding specificity involved in a number of important biological processes, such as neural wiring and innate immunity. Combinatorial use of alternatively spliced Ig-domains enables the generation of an estimated 18,000 isoform-specific homophilic receptor pairs. Although isoform diversity of Dscam is unique to arthropods, recent genetic analysis of vertebrate DSCAM (Down Syndrome Cell Adhesion Molecule) genes has revealed an intriguing conservation of molecular functions underlying neural wiring. This review covers the multiple functions of Dscam across different species highlighting its remarkable versatility as well as its conserved basic functions in neural development. We discuss how an unprecedented expansion of complex alternative splicing has been uniquely employed by arthropods to generate diverse surface receptors, important for cell-cell communication, molecular self-recognition in neurons, and innate immune defenses. We end with a speculative hypothesis reconciling the striking differences in Dscam and DSCAM gene structures with their conserved functions in molecular recognition underlying neural circuit formation.

Cell surface receptors in eukaryotes (some of which can be recognized in protozoan genomes) are thought to originate from simple cell adhesion molecules (CAMs) used as a glue during colony formation or from membrane proteins essential for prey recognition (King et al. 2003). Either way, the use of surface receptors to determine

[Keywords: Dscam; immunoglobulin receptor; alternative splicing; neural circuit formation]

${ }^{5}$ Corresponding author.

E-MAIL dietmar_schmucker@dfci.harvard.edu; FAX (617) 632-6499.

Article is online at http://www.genesdev.org/cgi/doi/10.1101/gad.1752909. molecular identity and signal "friend" versus "foe" expanded dramatically in metazoans where these CAMs now exert essential functions driving the evolutionary sophistication of complex tissues and immune defense systems. Not only have these molecular families grown, but they also have assumed additional roles to allow for novel functions in different tissues across development and in the adult (Boulanger et al. 2001).

Given the biological complexity of higher vertebrates, it is generally assumed that mammalian genes, especially those involved in nervous system development, are more numerous and more complex than invertebrate genes. This is surprisingly not the case for the Ig receptor Dscam, which is significantly more complex in arthropods. Even more perplexing are recent findings, which reveal that, despite the striking molecular differences, insect Dscam and mammalian DSCAM (Down Syndrome Cell Adhesion Molecule) share important functional roles in neural wiring. Uncovering the "core" molecular mechanisms of Dscam function in different organisms and systems promises to reveal key principles of how CAMs evolved into recognition units required for neural wiring and immune recognition or both.

DSCAM was first identified in an effort to characterize proteins located within human chromosome band 21q22, a region known to play a critical role in Down Syndrome (Yamakawa et al. 1998). The name Down Syndrome Cell Adhesion Molecule was chosen because of the chromosomal location, its appropriate expression in developing neural tissue, and its structure as an Ig receptor related to other CAMs. The immunoglobulin (Ig) superfamily is the largest group of related cell surface proteins, and is named after their extracellular cysteine-looped domains, originally described in the Ig proteins of the immune recognition system (Chothia et al. 1988; Williams and Barclay 1988; Harpaz and Chothia 1994). DSCAM proteins are one of the largest Ig superfamily CAMs discovered (220 $\mathrm{kDa})$, containing $10 \mathrm{Ig}$ domains and six fibronectin type III (FN) repeats, with the tenth Ig domain located between FN four and five (Fig. 1). The DSCAM protein structure is 
conserved with more than roughly $20 \%$ amino acid identity across the deuterostomes and protostomes, and assuming an ancestral homologous gene (Crayton et al. 2006; Brites et al. 2008) places the origin of the DSCAM gene at >600 million years ago. Since then, the DSCAM gene has been duplicated at least once in vertebrates and insects (Crayton et al. 2006; Brites et al. 2008). The second human DSCAM gene, DSCAML1, is located on chromosome band 11q23, a locus associated with Gilles de la Tourette and Jacobsen syndromes (Agarwala 2001). However, the most intriguing changes in gene structure have occurred in arthropods where several duplications of exons generated three large tandem arrays that are alternatively spliced (Schmucker et al. 2000). This alternative splicing of individual exon sequences within an array occurs in a mutually exclusive and combinatorial manner allowing for expression of tens of thousands of Dscam isoforms (Fig. 1). In the arthropods genomes these three large exon arrays encode the $\mathrm{N}$-terminal halves of the second and third Ig domains and the full Ig7 domain (red, blue, and green, respectively, in Fig. 1; Schmucker et al. 2000; Graveley et al. 2004; Watson et al. 2005; Brites et al. 2008).

\section{Mechanisms and molecular control of Dscam's alternative splicing}

The alternative splicing of Dscam is remarkable considering not only the resulting protein diversity but also its unique mechanism (one might say sophistication) of splicing. For example, the Dscam exon 6 cluster contains 48 alternative exon sequences in Drosophila mela- nogaster, yet, without exception, only a single exon 6 variant is included in a functional mRNA. Inclusion of two or more exon 6 sequences, or skipping of exon 6 would render the protein nonfunctional. Such a strict mode of exclusively selecting a single exon variant from dozens of equivalent tandemly arrayed sequences does not appear to exist in vertebrates. In this context, two mechanistic questions stand out: First, what accounts for the fidelity of single exon selection, and second, is the alternative exon selection random or not?

It appears that different mechanisms control the exclusive alternative splicing of exon 4 , exon 6 , and exon 9 clusters. The question of single exon selection in splicing of exon 4 and exon 6 variants was investigated by the Graveley group (Graveley 2005; Kreahling and Graveley 2005). They found evidence that the formation of RNA secondary structures is crucially involved in controlling splicing of both exon clusters, albeit in very different ways. For exon 4, an intronic sequence upstream of the exon 4 variants forms a stem/loop secondary structure (termed inclusion stem, or iStem). Deletion of this sequence causes exon 4 skipping in cell culture, but the iStem is not likely to have an influence on the choice of exon 4 variant (Kreahling and Graveley 2005). Moreover, it is still unknown how this secondary structure interacts with the splicing complex.

A comparative genomics study by Graveley (2005) has revealed the existence of highly conserved intronic sequences in the exon 6 complex, thought to enable the formation of competing intronic RNA secondary structures. A highly conserved sequence element termed
Figure 1. Domain architecture of the Ig receptor Dscam is conserved over 600 million years across invertebrate and vertebrate species. Phylogenetic analysis of Dscam gene and protein structure indicates a presumptive origin of Dscam before the split of the Bilaterians. Dscam protein has nine Ig domains from $\mathrm{N}$ terminus, six FN domains, with a single Ig domain between FN domains four and five. The most notable difference between the arthropod and vertebrate genomic organizations is the hypervariable exon arrays in arthropods that can generate tens of thousands of Dscam isoforms (see text). Alternative splicing of these exon array sequences (red, blue, and green) generate different sequences of the N-terminal half of Ig2 (red), the $\mathrm{N}$-terminal half of Ig3 (blue), and the full Ig7 domain (green). Remarkably, alternative splicing of these specific hypervariable Ig domains is conserved between crustaceans and insects, with a common ancestor $>400$ million years ago. It is not known whether the annelid and arachnid genomes contain Dscam genes (?); the nematode genomes currently available do not contain Dscam (ø). Data adapted from Yamakawa et al. 1998; Schmucker et al. 2000; Agarwala 2001; Graveley et al. 2004; Watson et al. 2005; Crayton et al. 2006; Fusaoka et al. 2006; Brites et al. 2008; and D. Schmucker, unpubl.

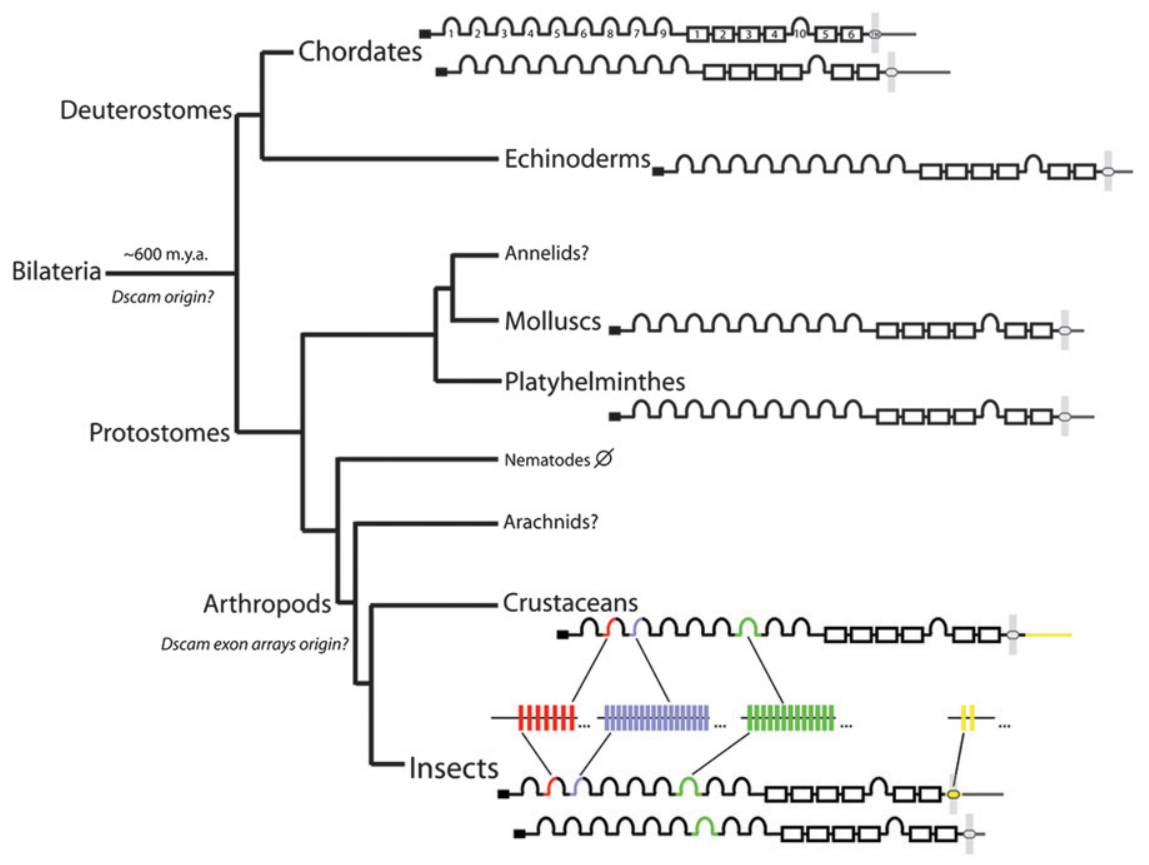


"docking site" 66 nucleotides in Drosophila melanogaster) is located downstream from the constant exon 5 and upstream of the variable exon 6 cluster. Shorter sequences termed "selector sequences" that are conspicuously complementary to sequences of the docking site are located upstream of each of the alternative exon 6 variants (Graveley 2005). Although the functional importance of these sequences has yet to be tested experimentally, the strong evolutionary conservation provides good support for a model in which base-pairing of docking and selector sites enables juxtaposition of a splice acceptor sequence of a single exon 6 sequence (exon 6.x) to the splice donor of constant exon 5. Presumably the intervening exon 6 variants are looped out and will be fully removed by the joining of exon 5 and exon 6.x in a first splicing step. To safeguard the next splicing step, the existence of a constitutive splicing repressor has been proposed to block inclusion of any exon 6 variants downstream from exon 6.x, while not interfering with the joining of exon 6.x and constant exon 7. In fact, such a splicing repressor, the heterogeneous nuclear ribonucleoprotein hrp36, has been shown to bind throughout the exon 6 cluster (Olson et al. 2007). Importantly, RNAimediated knockdown of hrp36 in Drosophila S2 cells resulted in aberrant inclusion of multiple exon 6 variants in spliced Dscam mRNAs without affecting alternative splicing in the other variable exon 4 and exon 9 clusters. This suggests that competitive base-pairing of intronic pre-mRNA sequences in combination with a general splicing repressor ensures mutually exclusive splicing of exon 6 sequences. In contrast, alternative splicing of exon 9 variants remains mysterious, as neither conserved sequence elements or specific splicing factors have yet been identified. This is unfortunate, as the splicing of exon 9 in particular shows a strong and intriguing bias in cell-type-specific splicing (discussed below).

The next and still largely unresolved question concerns whether it matters which one of the alternative exon sequences are chosen. This question was addressed by PCR-based expression analysis of same-type neurons or single cells (Hummel et al. 2003; Neves et al. 2004; Zhan et al. 2004). These experiments showed that photoreceptor, olfactory, or mushroom body neurons express broad yet distinctive sets of Dscam splice forms. Moreover, single-cell analysis revealed that single neurons or single Drosophila S2 cells (a hemocyte-like cell type) express more than one splice form. Based on these findings it was suggested that single neurons express at least a few and sometimes up to 50 different mRNA isoforms. It is important to note that no study has yet compared the Dscam isoform expression between individual animals focusing on a specific neuron at a specific developmental time. However, similar neurons of the same animal (e.g., developing outer photoreceptor cell R7 or inner photoreceptor cells R3/4) express different mRNAs without an apparent pattern of exon 4 or exon 6 choice, but with a significant restriction of exon 9 choice. Exon 9 splicing is also temporally regulated, with only a few exon 9 sequences expressed in the early embryo and the remaining exon 9 sequences become more prevalent with age.
Although these studies found evidence that the choice of splice variants is spatially and temporally regulated, the extent of this regulation for specific cells and specific developmental time points is currently not known. More importantly, it is not known whether a bias in alternative splicing is at all relevant for in vivo functions. One particularly interesting possibility has been suggested for a function of Dscam isoforms in the immune system. This remarkable study discovered that the alternative splicing of Dscam in the mosquito (Anopheles gambiae) immune system changes in response to various immune challenges (Dong et al. 2006). Moreover, the changes in alternative splicing do not appear to be random, and the choices of alternative exon selections are distinctly different depending on the pathogen used for the immune challenges. This was shown by quantitative PCR comparing the exon 4 choice in immune competent Sua5B cells that were either subjected to bacterial challenges or not. Consistent with changes in alternative splicing was the amount of Dscam specifically bound to Grampositive or Gram-negative bacteria increased if immune competent Sua5B cells had first been challenged with the respective bacteria, suggesting that the bacterial challenge increased the production of isoforms that have preferential affinity to the pathogen. These results provoke the speculation that this change in alternative splicing reflects an insect-specific form of adaptive immune response. However, many more experiments will be needed to examine such a possibility. As such, it will be necessary to determine whether distinct Dscam isoforms recognize specific epitopes on bacterial surfaces or not. In addition, more has to be learned about the in vivo importance and precise function of Dscam in immune responses in insects. Last but not least, it remains a puzzle how a specific recognition of a pathogenic antigen by one Dscam isoform may lead to changes in alternative splicing or splice form production of that same isoform.

\section{Homophilic binding and isoform specificity}

Many Ig superfamily molecules bind homophilically and heterophilically, and Dscam/DSCAM proteins are no exception. Vertebrate DSCAMs and DSCAML1s have not only been shown to bind homophilically (i.e., DSCAM-DSCAM or DSCAML1-DSCAML1, and not DSCAM-DSCAML1) (Agarwala et al. 2000; Yamagata and Sanes 2008), but also have cell-type-specific, mutually exclusive expression patterns (Barlow et al. 2002; Yamagata and Sanes 2008) (see below). Due to the combinatorial use of alternative exons the homophilic binding specificity of Drosophila Dscam is amplified to tens of thousands of potential homodimers (Wojtowicz 2004; Wojtowicz et al. 2007). Biochemical assays, cell-tocell and bead-to-cell binding assays were used to demonstrate that isoform-specific homodimerization occurs with remarkable binding specificity. Strong homophilic binding requires a minimum of seven $\mathrm{N}$-terminal Ig domains including all three variable Ig domains and involves direct binding of the matching Ig domains, pairing $\operatorname{Ig} 2$ with $\operatorname{Ig} 2$, Ig3 with $\operatorname{Ig} 3$, and $\operatorname{Ig} 7$ with $\operatorname{Ig} 7$ (Fig. 2). This 
Figure 2. Principles of isoform-specific homophilic Dscam receptor interactions. (A) Protein domain architecture of Dscam receptor isoforms, $\mathrm{N}$ terminus left side. Three of the first nine Ig domains (Ig2, Ig3, and $\operatorname{Ig} 7)$ contain amino acid sequences that, due to alternative splicing, differ between isoforms (see Fig. 1). These three "variable" Ig domains determine the homophilic binding specificity of the thousands of Dscam isoforms (Meijers et al. 2007; Wojtowicz et al. 2007). Differently colored ovals and logos indicate location and orientation of variable amino acid sequences. For simplicity, only part of the first fibronectin domain (FN1) is shown. The full extracellular part of Dscam contains additional FN domains (six in total) and one additional Ig (Ig10) domain. (B) Singlemolecule EM data indicate significant flexibility of Dscam extracellular domains (Meijers et al. 2007). However, intramolecular interactions stabilize a horseshoe configuration of the first four Ig domains. Such a horseshoe configuration leads to a juxtaposition of variable residues within Ig2 and Ig3 and a composite isoform-specific surface binding area termed "epitope 1." Among many other potential topologies, a particularly strong bend between the $\operatorname{Ig} 5$ and $\operatorname{Ig} 6$ domains (enabling an S-shape) appears to be necessary for achieving a configuration that can engage in dimer formation (Sawaya et al. 2008). Importantly, dimer formation requires direct matching interactions of all three variable Ig domains (Woitowicz et al. 2004). For all three variable domains, direct binding is mediated by short peptides specific for each Ig2, Ig3, and Ig7 domains (Meijers et al. 2007; Sawaya et al. 2008; further detailed discussions in Shapiro 2007 and Schmucker 2007). Biochemical data show that stable homo-dimer formation requires interactions of all three variable domains (Wojtowicz et al. 2004). However, it remains unknown whether separate lower-affinity interactions between two receptors with matching epitope 1 alone, or matching Ig7 alone, may occur in vivo.

was rigorously tested in a study using a high-throughput ELISA-based binding assay. Testing $>3000$ isoform pairs, specific homophilic binding was observed for all isoform combinations in which the three variable domains matched and only in a few exceptions was heterophilic binding observed (Wojtowicz et al. 2007). This reveals that Dscam diversity can give rise to $>18,000$ distinct homodimers.

\section{Molecular mechanism of homophilic binding specificity}

Two structural studies have investigated how such an enormous binding specificity could be achieved (Meijers et al. 2007; Sawaya et al. 2008). High-resolution crystal structures of ectodomain fragments containing the first four Ig domains (i.e., Ig1-4) of two different isoforms provided decisive insights in how variable domain interactions contribute to binding specificity (Fig. 2). Based on the exon 4 and exon 6 combination the Ig1-4 Dscam structures were designated D1-D4 $4_{1.34}$ and D1-D49.9. Comparing D1-D4 ${ }_{1.34}$ and D1-D49.9 reveals that despite the sequence differences, they nevertheless engage in highly similar dimer configurations (for review, see Schmucker 2007; Shapiro 2007). Importantly, isoformspecific residues encoded by alternative exon 4 and exon 6 are exposed on the surface of a horseshoe shaped $\mathrm{N}$ terminus where they constitute two distinct recognition sites, termed "epitope I" and "epitope II," positioned on each "face" of the receptor surface. Dimerization and thereby homophilic binding is only mediated through one binding surface, the epitope I surface. Residues that are unique among alternatively spliced exons within each of the variable Ig domains are directly involved in binding. The binding interface is formed by short peptide segments encoded by the variable $\mathrm{N}$-terminal half of exon 4 and exon 6 in both isoforms examined. These peptide segments pair in anti-parallel orientation and provide the specificity elements controlling homophilic interactions. Swapping the sequence segment containing epitope I but not epitope II results in a full switch in binding specificity between two isoforms, confirming that only epitope I is important for homophilic binding (Meijers et al. 2007).

Building on the atomic resolution structures of D1D4 ${ }_{1.34}$ and D1-D4 9.9 a systematic protein modeling of other variable Ig domains has been undertaken. Based on this and consistent with the biochemical data it became apparent that the principle of anti-parallel peptide pairing may apply to all Ig2-Ig2 and Ig3-Ig3 combinations (Wojtowicz et al. 2007). But does it also apply to $\operatorname{Ig} 7-\operatorname{Ig} 7$ interactions? This question has been addressed in a recent structural study on a larger Dscam ectodomain D1-

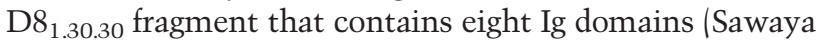
et al. 2008). A molecular replacement strategy based on the D1-D4 ${ }_{1.34}$ structure (Meijers et al. 2007) combined with selenomethionine-labeling allowed the building of a $4.2 \AA$ resolution model of the large and flexible D1-D8 $8_{1.30 .30}$ molecule. This structural model resolved the question of how $\operatorname{Ig} 7$ contributes to isoform-specific dimer formation. 
Analogous to the Ig2 and Ig3 domains, the D1-D8 1.30 .30 dimer also includes direct noncovalent contacts between the most variable residues of $\operatorname{Ig} 7$ molecules, which are paired in anti-parallel orientation within a dimer unit (Fig. 2). Binding involves only one face of one sheet of the Ig-characteristic $\beta$-sandwich. A strong intramolecular bend at the hinge of $\operatorname{Ig} 5-\operatorname{Ig} 6$ is essential for enabling an anti-parallel pairing of the Ig7 domains. Therefore, sharp intramolecular turns between Ig2 and Ig3 as well as between constant domains of $\operatorname{Ig} 5-\operatorname{Ig} 6$ enable an S-shaped confirmation of Dscam receptors (Fig. 2), which is likely to be essential for the formation of stable dimers.

Single-molecule electron microscopy (EM) studies (Meijers et al. 2007) together with thermodynamic considerations suggest that the Ig2-Ig3 turn is part of a constant horseshoe conformation, whereas the Ig5-Ig6 turn may only form upon dimerization (Sawaya et al. 2008). It seems likely that homophilic isoform interactions of Dscam receptors involve two distinct binding steps (Fig. 2). In one step, epitope I formed by a unique Ig2-Ig3 combination in one monomer has to be matched with an identical epitope I surface of another monomer (from 576 different variants). In another step, matching of identical Ig7 domains (33 different variants) has to be achieved, likely requiring a conformational stabilization of the Ig5-Ig6 turn. For the homophilic binding of full receptors, both binding steps are required, but it is currently unknown whether and how the two binding steps are coordinated. It is also unclear whether potential one-step "subthreshold" interactions (e.g., through separate horseshoe or Ig7 interactions) may have any cellular and in vivo consequences. However, due to the apparent flexibility of the Ig5-Ig6 hinge, it has been proposed that conformational changes involving Ig5 and Ig6 domains may allow for the regulatory control of dimer formation (Sawaya et al. 2008).

Taken together, these structural models provide a satisfying answer to the perplexing homophilic binding specificity of some 18,000 Dscam dimers. Consistent with the modularity of domain interactions, the binding strength for each individual domain interface is low (Meijers et al. 2007; Sawaya et al. 2008). Because of these relatively weak interactions of single domains, stable dimers will only be formed when all three variable domains fully interact (Fig. 2). These features of the combinatorial domain usage are essential for the generation of the extraordinarily large number of distinct homophilic binding pairs.

\section{Dscam is required during different steps of neural circuit formation}

The first functional characterization of Dscam demonstrated a role in axonal tract formation in the embryonic fly nervous system, and in axon guidance of the Bolwig's nerve of the larval visual system (Schmucker et al. 2000). The expression of Dscam remains mostly restricted to neural tissue where the protein is concentrated at axons and dendrites (Schmucker et al. 2000; Wang et al. 2004). Dscam-null animals die during larval stages and the early embryonic axonal patterning of the fly nervous system is clearly disrupted. In the absence of Dscam protein, Bolwig's axons exhibit frequent axon pathfinding errors at a specific intermediate target (Schmucker et al. 2000). In the planaria (Dugesia japonicum), Dscam is also required for the proper formation of axonal tracts during nervous system regeneration (Fusaoka et al. 2006). RNAimediated reduction of Dscam within newly generated flatworm brains results in highly disorganized axonal tracts, defects comparable with those seen in the developing embryonic nervous system of Dscam-null fly embryos.

Loss of Dscam during early nervous system formation in both flatworms and flies results in irregularities in the thickness of longitudinal axonal tracts, fasciculation defects, aberrant and ectopic branches, and a decrease in the number of lateral branches. However, in planaria, the loss of lateral axonal branches is due primarily to defects in cell migration, an additional role that many Ig superfamily members serve (Fusaoka et al. 2006). For example, the Ig superfamily molecule UNC-40 (an ortholog of DCC, the vertebrate netrin-1 receptor) has been shown to enable proper cell migration in the nematode Caenorhabditis elegans (Hedgecock 1990; Chan et al. 1996). Because Dscam is maternally expressed in early Zebrafish embryos (Yimlamai et al. 2005), Dscam has also been implicated in cell migration of embryonic cephalic cells destined to become neuroectoderm (Yimlamai et al. 2005).

DSCAM is also involved in axon guidance during vertebrate embryonic development, where it is required for the proper growth and turning of axons in the spinal cord as they turn ventrally to cross the midline floor plate (Ly et al. 2008). Floor plate cells are intermediate targets for spinal cord commissural axons and secrete a chemoattractant netrin-1 to guide axons toward them (Kennedy et al. 1994; Serafini et al. 1994). Stein and colleagues (Ly et al. 2008) have shown recently that netrin-1 is not only detected by the known netrin receptor DCC, but also by DSCAM. Biochemical experiments showed that DSCAM can bind netrin and that endogenous DSCAM receptors are found in a DCC-DSCAM complex. Activation of netrin-1 receptors in dorsal spinal cord explants induces growth of commissural axons and is dependent on both DCC and DSCAM. However, the directed turning of the axons toward the netrin-1 source in explant cultures was independent of DCC and mediated by DSCAM (Ly et al. 2008). These findings provide evidence that DSCAM can function not only as a homophilic receptor but also as a bona fide netrin receptor (Fig. 3). Therefore, depending on the cellular and developmental context, DSCAM can mediate netrin responses independent of DCC or cooperatively. Although it had been shown that in insect hemocytes, Dscam has an important function in binding heterophilic ligands such as bacterial surfaces (Watson et al. 2005), this study was the first to demonstrate a heterophilic ligand-binding activity of DSCAM in the nervous system. It is also interesting to note that the netrin ligand appears to bind to DSCAM in a region encompassing $\operatorname{Ig} 7-\operatorname{Ig} 9$. Given that in flies the $\operatorname{Ig} 7$ domain 
is crucial for homophilic binding (Fig. 2), this suggests the possibility that netrin binding may interfere with homophilic binding of DSCAM receptors (Fig. 3).

\section{Neuron branch segregation and in vivo function of Dscam homophilic repulsion}

Genetic analysis has provided good evidence that Dscam has important roles in neurite repulsion and branch formation. These functions clearly require homophilic binding of Dscam isoforms and they illustrate the broad importance of neurite self-avoidance for proper axonal and dendritic connectivity. The formation of axonal and dendritic fields is conceptualized as two very separate processes, but axons and dendrites both make extensive use of neurite branching during circuit formation.

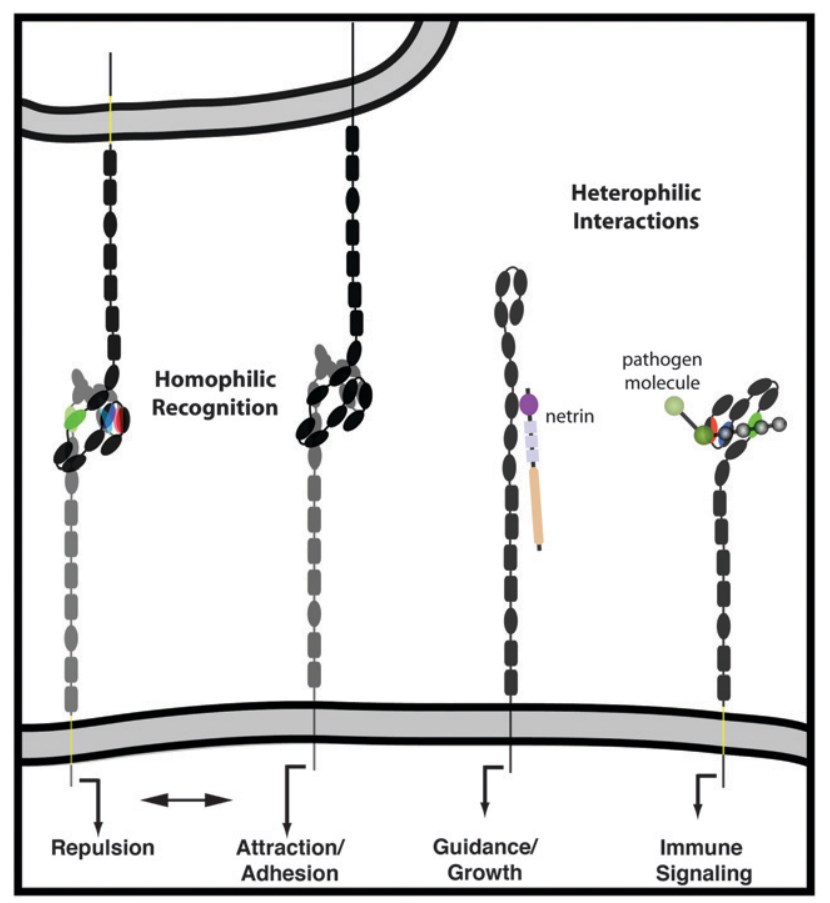

Figure 3. Dscam and DSCAM use homophilic and heterophilic interactions to initiate signaling underlying multiple functions. Isoform-specific homophilic binding of Drosophila Dscam (red, blue, and green Ig domains) enables an intrinsic selfrecognition for repulsion of same-cell axon (Wang et al. 2002; Zhan et al. 2004; Hattori et al. 2007) or dendrite (Hughes et al. 2007; Matthews et al. 2007; Soba et al. 2007) branches. Although mammalian DSCAM (monochrome receptors) lack diversity of isoforms, homophilic recognition is also important in mammalian receptors. Similar to other guidance receptors, in some biological contexts Dscam or DSCAM signaling might be switched to attraction rather than repulsion (Zhan et al. 2004; Yamagata and Sanes 2008). For example, homophilic recognition of vertebrate DSCAMs supports attraction/adhesion of neurites during synaptic targeting (Yamagata and Sanes 2008). Vertebrate DSCAM also binds netrin (i.e., heterophilic ligand) for axon guidance functions (Ly et al. 2008). Dscam's requirement in innate immune responses to pathogens suggests heterophilic binding to different pathogen molecules (Watson et al. 2005; Dong et al. 2006).

\section{Axon bifurcation}

During the formation of a nascent axonal branch it is crucial to ensure its proper separation from the main axon through a repulsive signal. In the absence of such a signal, the newly formed branch will fasciculate with the founder axon and continue to follow the same trajectory and guidance cues. Dscam was found to mediate axon branch repulsion in neurons of the mushroom body, a central brain structure of Drosophila (Wang et al. 2002; Zhan et al. 2004; Hattori et al. 2007). The mushroom body has two lobes that are formed by the bifurcation of thousands of axons into a medial and lateral axonal bundle (Ito et al. 1997). Loss of Dscam within these axons reduces the probability that an axonal branch will properly segregate into a second lobe, and instead remains traveling parallel with the founder branch into the first lobe (Wang et al. 2002; Zhan et al. 2004).

Because Drosophila Dscam can generate tens of thousands of different isoforms that exhibit homophilic binding specificity, its molecular diversity has been postulated to uniquely label neuronal processes. Consistent with this notion, genetic manipulations that eliminate Dscam isoform diversity and replace it with a single isoform result in the formation of a mushroom body containing only a single lobe (Hattori et al. 2007). In contrast, deletion mutants that reduced Dscam diversity into the thousands of isoforms range by removing all but one Ig2 variant produced still normal lobes and suggested that no specific subset was required for this process (Wang et al. 2002). Therefore, it seems that the segregation of thousands of axon branches into a secondary bundle requires an isoform diversity of more than one and possibly many but not the full repertoire of Dscam isoforms.

Within a single mushroom body axon, expression of any one isoform chosen will allow the splitting of an axon into two branches to enter the dual mushroom body lobes (Zhan et al. 2004; Hattori et al. 2007). Thus, in this simple axon bifurcation paradigm, Dscam function does not require specific isoform expression consistent with a hypothesis that isoforms are randomly expressed and that any isoform can mediate a homophilic repulsion response to separate nascent axon branches (Wang et al. 2002; Zhan et al. 2004; Hattori et al. 2007). Given the large number of possible isoforms, the probability that a nearby branch will express the same randomly chosen subset of isoforms is negligible, unless that branch comes from the same cell, in which case it would be successfully repelled into the second lobe due to the homophilic binding of a perfect match of Dscam isoforms. However, it is important to emphasize that this strictly noninstructive use of Dscam isoforms is not likely to apply to more complex axonal branching and targeting decisions (discussed below).

\section{Sister dendrite repulsion}

Neurons have very diverse, yet tightly regulated dendritic arbor morphologies, which are a hallmark of their neuronal identity and inseparably linked to their functions (Häusser et al. 2000; Landgraf and Evers 2005). For example, somatosensory neurons have broad, topographically 
restricted receptive fields with evenly spaced soma positions and dendrites, providing an even coverage of the sensory field. During segregation and spacing of dendritic branches, one possible mechanism of controlling the distances between branches is through the use of cell surface or diffusible repulsive cues. Dscam-mediated repulsion has been most intensely studied in the context of dendrite morphogenesis (Zhu et al. 2006; Hughes et al. 2007; Matthews et al. 2007; Soba et al. 2007) providing the best example for the in vivo importance of homophilic (and isoform-specific) recognition.

Dscam was first shown to be involved in the formation of dendritic trees in Drosophila olfactory projection neurons (Zhu et al. 2006). Loss of Dscam within projection neurons as well as in local interneurons resulted in reduced dendritic arbors and sometimes abnormal dendritic elaborations. Similar results were seen in the dendrites of Drosophila larval sensory neurons termed dendrite arborization (DA) neurons (Hughes et al. 2007; Matthews et al. 2007; Soba et al. 2007). Dendrites of DA neurons exhibit "self-avoidance" where the dendritic branches of the same neuron will tend to avoid each other to provide an even coverage of the neuron's receptive field space. Some classes of DA neurons (class IV neurons) evenly tile the body wall and avoid the dendritic branches of other neighboring DA neurons, but only of the same class (Grueber et al. 2002). In DA neurons, Dscam is required for dendritic self-avoidance, but not for avoidance of dendrites from other neurons within the same class.

Cell-autonomous loss of Dscam leads to frequent dendrite self-crossing and dendrite fasciculation, consistent with a repulsive function of Dscam. Expression of a single randomly chosen Dscam isoform is sufficient to rescue these self-crossing defects (Hughes et al. 2007; Matthews et al. 2007; Soba et al. 2007). Importantly, the Dscam-mediated repulsive response depends on the presence of a cytoplasmic signal-transducing domain of Dscam. Expression of a truncated Dscam receptor fails to rescue self-avoidance and leads to dominant phenotypes causing dendrites to clump and fasciculate together. Moreover, expression of a single Dscam isoform in two neurons of different classes, which normally elaborate overlapping dendritic fields, forces the dendritic trees of these neurons to segregate completely (Hughes et al. 2007; Matthews et al. 2007; Soba et al. 2007).

The genetic analysis of DA neuron morphogenesis as well as the axon bifurcation in mushroom body neurons are consistent with the following model: Homophilic binding of identical Dscam isoforms initiates a signaling cascade that converts homophilic recognition into neurite repulsion (Fig. 3). The isoform specificity allows restricting this repulsion only to interactions with "self" surfaces while "tolerating" contact with other neurites.

\section{Dscam's role in repulsive functions is conserved in mammalian DSCAM}

Given that the two vertebrate DSCAM genes are not extensively alternatively spliced allows for an analysis of the simplest case for homophilic repulsion, one mediated by a single Dscam isoform. A spontaneous mutation in the mouse Dscam gene discovered at Jackson Laboratories lead to the first loss-of-function analysis of mammalian DSCAM function. Mice homozygous for this hypomorphic DSCAM allele were found to exhibit several severe neurological defects such as ataxia and seizures (Fuerst et al. 2008). Analysis of subsets of amacrine cells in the retinas of these mice revealed abnormally positioned somas with fasciculated dendrites compared with regularly spaced soma positions and dendritic branches in wild-type mice. This phenotype is restricted to amacrine cells that normally express DSCAM. However, earlier in development, these amacrine cell bodies in the DSCAM mutant retina are evenly spaced, suggesting that DSCAM is required to maintain the position of these cells. This is most likely accomplished by promoting inhibitory interactions between retinal territories in DSCAM-expressing cells of the same class (Fuerst et al. 2008). Therefore, in the mouse retina, DSCAM receptors serve not only cell-intrinsic functions important for dendrite self-avoidance, but also dictate cell-cell interactions, which underlie migration and cell positioning in the retina. These functions of DSCAM in the mouse retina are strikingly similar to the repulsive functions of Dscam in flies.

However, to complicate things, an interesting switch of DSCAM function was recently found for targeting of axons and dendrites to specific laminas in the chick retina (Yamagata and Sanes 2008). In this biological context homophilic DSCAM interactions promote rather than repel neurite contacts. The synaptic laminas in the vertebrate retina can be subdivided into different sublayers where inputs converge and diverge onto various cell types (Wässle 2004). At least some synaptic partner matching within the sublaminas of the chick retina depend on matching expression of DSCAM and DSCAM-L1 as well as Sidekick proteins (Yamagata and Sanes 2008). Sidekick proteins are also Ig superfamily members closely related to the DCSAM subgroup. DSCAMs and Sidekicks are expressed in mutually exclusive subsets of retinal cells that each have specific lamination patterns. Misexpression of any one of the receptors shifts the laminar targeting of neurons to abnormally connect with recipient cells that normally express the identical receptor (Yamagata and Sanes 2008). Currently, this is the only example that suggests an instructive role of DSCAM in neural circuit formation, and likely reflects an attraction-mediated function of DSCAM (Fig. 3). It is likely that developmentally regulated and cell-specific changes of intracellular signaling (potentially also involving coreceptors) may account for such a switch of repulsive to attractive function of DSCAM, similar to what has been described for related neuronal receptors (Dickson et al. 2000).

\section{Noncanonical Dscam functions}

Synaptic target selection. The canonical "self-avoidance" role of Drosophila Dscam in axon bifurcation and sister dendrite repulsion suggests a purely cell-intrinsic function 
of Dscam. However, the molecularly diverse receptor also has diverse cellular functions. One important example was provided by Zipursky and colleagues (Hummel et al. 2003) examining the development of the Drosophila olfactory system. Drosophila olfactory receptor neurons (ORNs) send their axons from the antenna and maxillary palps into the antennal lobe to target specific structures called glomeruli. The targeting of a specific axon to a specific glomerulus correlates with the OR gene expression, yet, in contrast to the mammalian olfactory system, is not dependent on OR function (Dobritsa et al. 2003; Jefferis et al. 2004). Loss of Dscam selectively from ORNs causes numerous axon targeting mistakes in the majority of ORNs once they enter the antennal lobe neuropil and affects the axon guidance of some ORNs on their path from the maxillary pulp to the antennal lobe (Hummel et al. 2003). Dscam mutant ORN axons exhibit a complex series of phenotypes that includes targeting to the incorrect glomerulus, mistargeting within the ipsi- as well as contralateral antennal lobe, and formation of extra glomeruli. These studies showed that Dscam is required in ORNs to connect with the correct glomerulus, and it was therefore proposed that Dscam mediates interactions between growth cones of ORNs and potential target regions within the neuropil (Hummel et al. 2003). However, it is currently not known whether axonal targeting of ORNs requires diverse Dscam isoforms.

Control of complex branching. A profound requirement for isoform diversity during axon targeting decisions was shown using the complex axonal branching patterns of mechanosensory (MS) neurons located in the thorax of flies (Chen et al. 2006). Thoracic MS neurons are located peripherally and innervate external bristles, which sense airflow and touch. Afferent projections of MS neurons are complex and exhibit many axonal branches, each of which connect with different post-synaptic targets within the CNS. Axon branch number and spatial position is largely invariant between individual flies. Dscam was found to be essential for MS neurons in establishing connectivity within the CNS. MS neurons that lack all Dscam isoforms (Dscam-null) lack all discernable axonal branch connections with post-synaptic neurons, and stall close to the CNS entry site. Expression of single randomly chosen isoforms is able to restore axon growth within the CNS but fails to rescue normal axon branch connectivity. Importantly, even animals that have normal levels of Dscam protein, but a reduced alternative exon repertoire, significantly change branching and targeting of afferent projections. Homozygous mutant flies lacking different subsets of alternative exon 4 sequences but expressing normal levels of wild-type protein were used and projections of single MS neurons were examined. It was found that the vast majority of flies with reduced Dscam diversity ( $\sim 40 \%$ reduction) show axonal branching and targeting differences, which includes defects such as ectopic branches, incorrect contralateral versus ipsilateral targeting, absence of axon branches, as well as large increases in axon branch variability compared with wild type (Chen et al. 2006). Interestingly, distinctly different but also common phenotypes were observed by removing different but overlapping subsets of exon 4 variants, suggesting nonredundant and possibly specific functions for different isoforms. These finding raise many important questions and require a more complex interpretation of Dscam functions. It will be important to determine whether diverse isoforms are required solely within the MS neurons or their targets, or both. In any case, these studies provide strong evidence that a large number or possibly the entire repertoire of diverse Dscam isoforms is functionally required for the precision of complex axonal branching and connectivity in the CNS (Chen et al. 2006).

\section{Perspectives}

Dscam is an outstanding example of how molecular specificity of membrane receptors controls neural wiring. Genetic studies of Dscam and DSCAM functions emphasize the importance of self-recognition in neurons as a developmental principle underlying neural circuit formation. Alternative splicing in arthropods generates thousands of distinct receptor isoforms, which can serve as identity tags in neurons. The specificity in homophilic recognition allows cells to "read" these molecular tags. Neurites of the same cell use Dscam-mediated molecular identity recognition (homotypic recognition) in order to avoid isoneuronal contact, neurite overlap and possibly other aspects of neural wiring (Fig. 3). There can be little doubt that the principle of homotypic recognition is also an important molecular principle in at least some vertebrate neurons as well. The mosaic spacing of amacrine cells in the mammalian retina provides just one of many examples, and it is intriguing that it involves the nondiversified vertebrate DSCAM receptor.

But why is there such a discrepancy between vertebrate DSCAM and invertebrate Dscam genes? Why is there a complex gene in simpler animals, and a simple gene in more complex animals?

It has been suggested that in vertebrates, combinatorial use of receptors of moderately diverse gene families such as the cadherins, protocadherins, or the neurexins provides an alternate receptor system (Zipursky et al. 2006). This is an attractive hypothesis, and addresses the difficulty of accounting for a molecular diversity large enough to provide specificity for the extraordinary large number of neurons in the more complex vertebrate brains. However, given the striking functional similarities between Dscam and DSCAM, we would like to speculate that despite the absence of isoform diversity, vertebrate DSCAM, and DSCAML1 receptors maintained a functional role in complex molecular recognition processes complementing the functions of other CAMs and newly diversified receptors (e.g., Neurexins). We therefore propose the following hypothesis.

It seems possible that Dscam in flies as well as DSCAM receptors in vertebrates function as an integral part of a receptor complex, rather than alone. A key function of such a Dscam or DSCAM containing receptor complex would be to couple molecular recognition of homotypic versus heterotypic neurite interactions to intracellular 
signal transduction. In flies, different complexes would contain different Dscam isoforms, providing different recognition specificities. In vertebrates, different DSCAM complexes would contain different combinations of diverse coreceptors, coupling them to a conserved signal transducing function of the constant receptor component DSCAM. Even though DSCAM and DSCAML1 are unlikely expressed in all neurons, the broad neuronal expression (Barlow et al. 2002) is consistent with a proposed role in diverse recognition processes required for the development of many different neuronal cell types. In this speculative hypothesis we suggest that during vertebrate evolution the recognition specificity of diverse Dscam receptor isoforms was transferred to coreceptors while at least some signal transducing functions were conserved in vertebrate DSCAMs. While experimental scrutiny is needed to test this idea, it seems certain that comparative studies of Dscam and DSCAM will continue to provide new insights in the molecular control of neural circuit formation.

\section{References}

Agarwala, K. 2001. Cloning and functional characterization of DSCAML1, a novel DSCAM-like cell adhesion molecule that mediates homophilic intercellular adhesion. Biochem. Biophys. Res. Commun. 285: 760-772.

Agarwala, K.L., Nakamura, S., Tsutsumi, Y., and Yamakawa, K. 2000. Down syndrome cell adhesion molecule DSCAM mediates homophilic intercellular adhesion. Brain Res. Mol. Brain Res. 79: 118-126.

Barlow, G., Micales, B., Chen, X., Lyons, G.E., and Korenberg, J. 2002. Mammalian DSCAMs: Roles in the development of the spinal cord, cortex, and cerebellum? Biochem. Biophys. Res. Commun. 293: 881-891.

Boulanger, L.M., Huh, G.S., and Shatz, C.J. 2001. Neuronal plasticity and cellular immunity: Shared molecular mechanisms. Curr. Opin. Neurobiol. 11: 568-578.

Brites, D., McTaggart, S., Morris, K., Anderson, J., Thomas, K., Colson, I., Fabbro, T., Little, T.J., Ebert, D., and Du Pasquier, L. 2008. The Dscam homologue of the crustacean Daphnia is diversified by alternative splicing like in insects. Mol. Biol. Evol. 25: 1429-1439.

Chan, S.S., Zheng, H., Su, M.W., Wilk, R., Killeen, M.T., Hedgecock, E.M., and Culotti, J.G. 1996. UNC-40, a C. elegans homolog of DCC (deleted in colorectal cancer), is required in motile cells responding to UNC-6 netrin cues. Cell 87: 187-195.

Chen, B., Kondo, M., Garnier, A., Watson, F., Puettmannholgado, R., Lamar, D., and Schmucker, D. 2006. The molecular diversity of dscam is functionally required for neuronal wiring specificity in Drosophila. Cell 125: 607-620.

Chothia, C., Boswell, D.R., and Lesk, A.M. 1988. The outline structure of the T-cell $\alpha \beta$ receptor. EMBO J. 7: 3745-3755.

Crayton, M.E., Powell, B.C., Vision, T.J., and Giddings, M.C. 2006. Tracking the evolution of alternatively spliced exons within the Dscam family. BMC Evol. Biol. 6: 16. doi:10.1186/ 1471-2148-6-16.

Dickson, B.J. 2000. Molecular mechanisms of axon guidance. Science 298: 1959-1964.

Dobritsa, A.A., van der Goes van Naters, W., Warr, C.G., Steinbrecht, R.A., and Carlson, J.R. 2003. Integrating the molecular and cellular basis of odor coding in the Drosophila antenna. Neuron 37: 827-841.
Dong, Y., Taylor, H., and Dimopoulos, G. 2006. AgDscam, a hypervariable immunoglobulin domain-containing receptor of the anopheles gambiae innate immune system. PLOS Biol. 4: e229. doi:10.1371/journal.pbio.0040229.

Fuerst, P., Koizumi, A., Masland, R., and Burgess, R. 2008. Neurite arborization and mosaic spacing in the mouse retina require DSCAM. Nature 451: 470-474.

Fusaoka, E., Inoue, T., Mineta, K., Agata, K., and Takeuchi, K. 2006. Structure and function of primitive immunoglobulin superfamily neural cell adhesion molecules: A lesson from studies on planarian. Genes Cells 11: 541-555.

Graveley, B. 2005. Mutually exclusive splicing of the insect premRNA directed by competing intronic RNA secondary structures. Cell 123: 65-73.

Graveley, B., Kaur, A., Gunning, D., Zipursky, S., Rowen, L., and Clemens, J. 2004. The organization and evolution of the dipteran and hymenopteran Down syndrome cell adhesion molecule (Dscam) genes. RNA 10: 1499-1506.

Grueber, W.B., Jan, L.Y., and Jan, Y.N. 2002. Tiling of the Drosophila epidermis by multidendritic sensory neurons. Development 129: 2867-2878.

Harpaz, Y. and Chothia, C. 1994. Many of the immunoglobulin superfamily domains in cell adhesion molecules and surface receptors belong to a new structural set which is close to that containing variable domains. J. Mol. Biol. 238: 528539.

Hattori, D., Demir, E., Kim, H., Viragh, E., Zipursky, S., and Dickson, B. 2007. Dscam diversity is essential for neuronal wiring and self-recognition. Nature 449: 223-227.

Häusser, M., Spruston, N., and Stuart, G.J. 2000. Diversity and dynamics of dendritic signaling. Science 290: 739-744.

Hedgecock, E. 1990. The unc-5, unc-6, and unc-40 genes guide circumferential migrations of pioneer axons and mesodermal cells on the epidermis in C. elegans. Neuron 4: 61-85.

Hughes, M., Bortnick, R., Tsubouchi, A., Baumer, P., Kondo, M., Uemura, T., and Schmucker, D. 2007. Homophilic Dscam interactions control complex dendrite morphogenesis. Neuron 54: 417-427.

Hummel, T., Vasconcelos, M.L., Clemens, J., Fishilevich, Y., Vosshall, L.B., and Zipursky, S. 2003. Axonal targeting of olfactory receptor neurons in Drosophila is controlled by Dscam. Neuron 37: 221-231.

Ito, K., Awano, W., Suzuki, K., Hiromi, Y., and Yamamoto, D. 1997. The Drosophila mushroom body is a quadruple structure of clonal units each of which contains a virtually identical set of neurones and glial cells. Development 124: 761-771.

Jefferis, G.S.X.E., Vyas, R.M., Berdnik, D., Ramaekers, A., Stocker, R.F., Tanaka, N.K., Ito, K., and Luo, L. 2004. Developmental origin of wiring specificity in the olfactory system of Drosophila. Development 131: 117-130.

Kennedy, T.E., Serafini, T., de la Torre, J.R., and Tessier-Lavigne, M. 1994. Netrins are diffusible chemotropic factors for commissural axons in the embryonic spinal cord. Cell 78: 425-435.

King, N., Hittinger, C.T., and Carroll, S.B. 2003. Evolution of key cell signaling and adhesion protein families predates animal origins. Science 301: 361-363.

Kreahling, J.M. and Graveley, B. 2005. The iStem, a long-range RNA secondary structure element required for efficient exon inclusion in the Drosophila Dscam pre-mRNA. Mol. Cell. Biol. 25: 10251-10260.

Landgraf, M. and Evers, J.F. 2005. Control of dendritic diversity. Curr. Opin. Cell Biol. 17: 690-696.

Ly, A., Nikolaev, A., Suresh, G., Zheng, Y., Tessier-Lavigne, M., and Stein, E. 2008. DSCAM is a netrin receptor that 
collaborates with DCC in mediating turning responses to netrin-1. Cell 133: 1241-1254.

Matthews, B., Kim, M., Flanagan, J., Hattori, D., Clemens, J., Zipursky, S., and Grueber, W. 2007. Dendrite self-avoidance is controlled by Dscam. Cell 129: 593-604.

Meijers, R., Puettmann-Holgado, R., Skiniotis, G., Liu, J., Walz, T., Wang, J., and Schmucker, D. 2007. Structural basis of Dscam isoform specificity. Nature 449: 487-491.

Neves, G., Zucker, J., Daly, M., and Chess, A. 2004. Stochastic yet biased expression of multiple Dscam splice variants by individual cells. Nat. Genet. 36: 240-246.

Olson, S., Blanchette, M., Park, J., Savva, Y., Yeo, G., Yeakley, J., Rio, D., and Graveley, B. 2007. A regulator of Dscam mutually exclusive splicing fidelity. Nat. Struct. Mol. Biol. 14: 1134-1140.

Sawaya, M.R., Wojtowicz, W.M., Andre, I., Qian, B., Wu, W., Baker, D., Eisenberg, D., and Zipursky, S. 2008. A double $S$ shape provides the structural basis for the extraordinary binding specificity of Dscam isoforms. Cell 134: 1007-1018.

Schmucker, D. 2007. Molecular diversity of Dscam: Recognition of molecular identity in neuronal wiring. Nat. Rev. Neurosci. 8: 915-920.

Schmucker, D., Clemens, J.C., Shu, H., Worby, C.A., Xiao, J., Muda, M., Dixon, J.E., and Zipursky, S.L. 2000. Drosophila Dscam is an axon guidance receptor exhibiting extraordinary molecular diversity. Cell 101: 671-684.

Serafini, T., Kennedy, T.E., Galko, M.J., Mirzayan, C., Jessell, T.M., and Tessier-Lavigne, M. 1994. The netrins define a family of axon outgrowth-promoting proteins homologous to C. elegans UNC-6. Cell 78: 409-424.

Shapiro, L. 2007. Self-recognition at the atomic level: Understanding the astonishing molecular diversity of homophilic Dscams. Neuron 56: 10-13.

Soba, P., Zhu, S., Emoto, K., Younger, S., Yang, S., Yu, H., Lee, T., Jan, L., and Jan, Y. 2007. Drosophila sensory neurons require dscam for dendritic self-avoidance and proper dendritic field organization. Neuron 54: 403-416.

Wang, J., Zugates, C., Liang, I.H., Lee, C.H., and Lee, T. 2002. Drosophila Dscam is required for divergent segregation of sister branches and suppresses ectopic bifurcation of axons. Neuron 33: 559-571.

Wang, J., Ma, X., Yang, J., Zheng, X., Zugates, C., Lee, C., and Lee, T. 2004. Transmembrane/juxtamembrane domaindependent Dscam distribution and function during mushroom body neuronal morphogenesis. Neuron 43: 663-672.

Wässle, H. 2004. Parallel processing in the mammalian retina. Nat. Rev. Neurosci. 5: 747-757.

Watson, F.L., Püttmann-Holgado, R., Thomas, F., Lamar, D.L., Hughes, M., Kondo, M., Rebel, V.I., and Schmucker, D. 2005. Extensive diversity of Ig-superfamily proteins in the immune system of insects. Science 309: 1874-1878.

Williams, A.F. and Barclay, A.N. 1988. The immunoglobulin superfamily-Domains for cell surface recognition. Annu. Rev. Immunol. 6: 381-405.

Wojtowicz, W. 2004. Alternative splicing of Drosophila Dscam generates axon guidance receptors that exhibit isoformspecific homophilic binding. Cell 118: 619-633.

Wojtowicz, W., Wu, W., Andre, I., Qian, B., Baker, D., and Zipursky, S. 2007. A vast repertoire of Dscam binding specificities arises from modular interactions of variable Ig domains. Cell 130: 1134-1145.

Yamagata, M. and Sanes, J. 2008. Dscam and Sidekick proteins direct lamina-specific synaptic connections in vertebrate retina. Nature 451: 465-469.

Yamakawa, K., Huot, Y.K., Haendelt, M.A., Hubert, R., Chen, X.N., Lyons, G.E., and Korenberg, J.R. 1998. DSCAM: A novel member of the immunoglobulin superfamily maps in a Down syndrome region and is involved in the development of the nervous system. Hum. Mol. Genet. 7: 227-237.

Yimlamai, D., Konnikova, L., Moss, L., and Jay, D. 2005. The zebrafish down syndrome cell adhesion molecule is involved in cell movement during embryogenesis. Dev. Biol. 279: 4457.

Zhan, X., Clemens, J., Neves, G., Hattori, D., Flanagan, J., Hummel, T., Vasconcelos, M., Chess, A., and Zipursky, S. 2004. Analysis of Dscam diversity in regulating axon guidance in mushroom bodies. Neuron 43: 673-686.

Zhu, H., Hummel, T., Clemens, J., Berdnik, D., Zipursky, S., and Luo, L. 2006. Dendritic patterning by Dscam and synaptic partner matching in the Drosophila antennal lobe. Nat. Neurosci. 9: 349-355.

Zipursky, S., Wojtowicz, W.M., and Hattori, D. 2006. Got diversity? Wiring the fly brain with Dscam. Trends Biochem. Sci. 31: 581-588. 


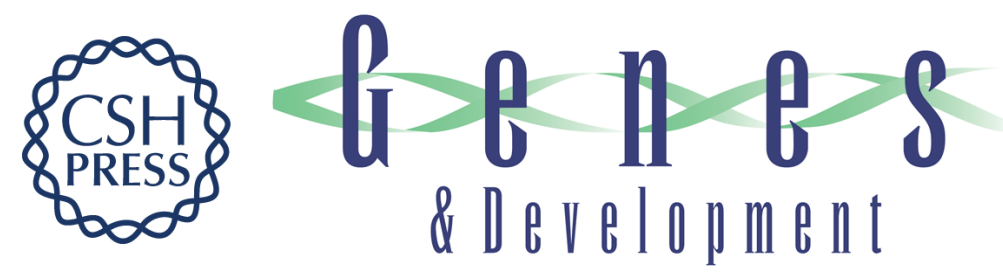

\section{Dscam and DSCAM: complex genes in simple animals, complex animals yet simple genes}

Dietmar Schmucker and Brian Chen

Genes Dev. 2009, 23:

Access the most recent version at doi:10.1101/gad.1752909

References This article cites 53 articles, 7 of which can be accessed free at: http://genesdev.cshlp.org/content/23/2/147.full.html\#ref-list-1

License

Email Alerting

Receive free email alerts when new articles cite this article - sign up in the box at the top Service right corner of the article or click here.

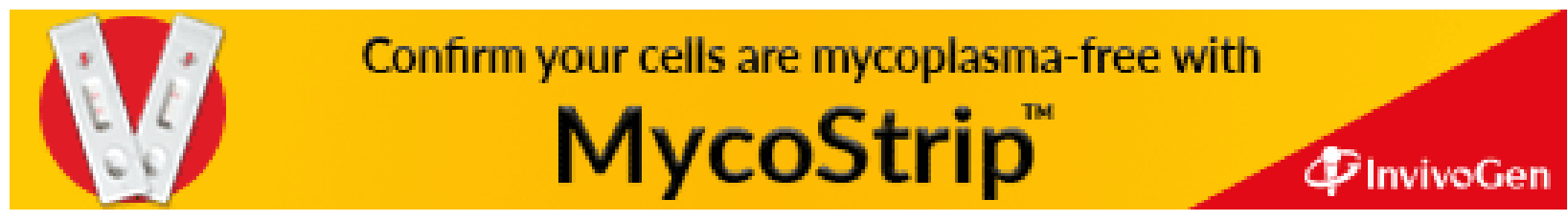

